\title{
Dual-source dual-energy computed tomography-derived quantitative parameters combined with machine learning for the differential diagnosis of benign and malignant thyroid nodules
}

\author{
Liling Jiang ${ }^{1,2 \#} \wedge$, Daihong Liu ${ }^{1,2 \#} \wedge$, Ling Long ${ }^{1,2} \wedge$, Jiao Chen $^{1,2}$, Xiaosong Lan $^{1,2} \wedge$, Jiuquan Zhang ${ }^{1,2} \wedge$ \\ ${ }^{1}$ Department of Radiology, Chongqing University Cancer Hospital, School of Medicine, Chongqing University, Chongqing, China; ${ }^{2}$ Chongqing \\ Cancer Multi-omics Big Data Application Engineering Research Center, Chongqing University Cancer Hospital, Chongqing, China
}

Contributions: (I) Conception and design: J Zhang; (II) Administrative support: L Jiang, D Liu; (III) Provision of study materials or patients: L Long, J Chen; (IV) Collection and assembly of data: L Jiang, J Chen; (V) Data analysis and interpretation: D Liu, X Lan; (VI) Manuscript writing: All authors; (VII) Final approval of manuscript: All authors.

"These authors contributed equally to this work.

Correspondence to: Prof. Jiuquan Zhang. Department of Radiology, Chongqing University Cancer Hospital, School of Medicine, Chongqing University, Chongqing 400030, China. Email: zhangjq_radiol@foxmail.com.

Background: This study aimed to investigate the ability of quantitative parameter-derived dual-source dual-energy computed tomography (DS-DECT) combined with machine learning to distinguish between benign and malignant thyroid nodules.

Methods: Patients with thyroid nodules and pathological surgical results who underwent preoperative DS-DECT were selected. Quantitative parameter-derived DS-DECT was applied to classify benign and malignant nodules. Then, machine learning and binary logistic regression analysis models were constructed using the DS-DECT quantitative parameters to distinguish between benign and malignant nodules. The receiver operating characteristic curve was used to assess the diagnostic performance. The DeLong test was used to compare the diagnostic efficacy.

Results: One hundred and thirty patients with 139 confirmed thyroid nodules were involved in the

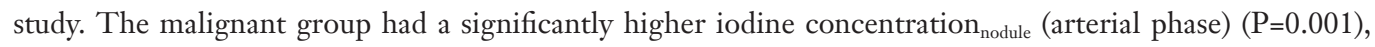
normalized iodine concentration (arterial phase) $(\mathrm{P}=0.002)$, iodine concentration difference $(\mathrm{P}<0.001)$, spectral curve slope (nonenhancement) $(\mathrm{P}=0.007)$, spectral curve slope (arterial phase) $(\mathrm{P}=0.001)$, effective atomic number (nonenhancement) $(\mathrm{P}<0.001)$, and effective atomic number (arterial phase) $(\mathrm{P}=0.039)$ than the benign group. The binary logistic regression analysis model had an AUC (area under the curve) of 0.76, a sensitivity of 0.821 , and a specificity of 0.667 . The machine learning model had an AUC of 0.86 , a sensitivity of 0.822 , specificity of 0.791 in the training cohort, an AUC of 0.84 , a sensitivity of 0.727 , and specificity of 0.750 in the testing cohort.

Conclusions: Multiple quantitative parameters of DS-DECT combined with machine learning could differentiate between benign and malignant thyroid nodules.

Keywords: Thyroid nodule; dual-source dual-energy computed tomography (DS-DECT); machine learning

Submitted May 09, 2021. Accepted for publication Aug 12, 2021.

doi: 10.21037/qims-21-501

View this article at: https://dx.doi.org/10.21037/qims-21-501

^ ORCID: Liling Jiang, 0000-0001-6457-3228; Daihong Liu, 0000-0001-5606-4394; Ling Long, 0000-0001-9265-5714; Jiao Chen, 00000001-7023-5073; Xiaosong Lan, 0000-0002-6199-5211; Jiuquan Zhang, 0000-0003-0239-6988. 


\section{Introduction}

With the increasing use of medical imaging for screening, diagnosis, and treatment response evaluation in recent decades, as many as $67 \%$ of thyroid incidentalomas are detected in the general population $(1,2)$. The malignancy rate of thyroid nodules is $10-15 \%$ (3). Differentiating malignant thyroid nodules from benign ones is important in planning further therapeutic approaches and the extent of surgical intervention.

Ultrasonography is the primary imaging test used to detect thyroid nodules, but it has some limitations in showing the relationship of the tumor to the surrounding structures and in identifying lymph node metastasis $(4,5)$. Fine-needle aspiration is an invasive examination, and one-third of the results are inconclusive (3). With the rapid development of magnetic resonance imaging, some functional sequences have achieved better diagnostic performance, but this method has the disadvantages of being high cost and time-consuming (6). Computed tomography (CT) can be used to identify nodules, establish preoperative localization, provide information for the diagnosis, and assess the therapeutic effect. However, conventional CT is limited in differentiating between benign and malignant thyroid nodules due to beam hardening artifacts and poor contrast with the surrounding thyroid tissue (7).

The recent technical advance of dual-source dualenergy CT (DS-DECT) has improved the situation, as this method uses two different X-ray sources, allowing for the simultaneous use of two different energies to achieve potential tissue differentiation. The advanced quantitative technique can separate several types of substances, such as water, iodine, calcium, uric acid, and fat (8-11). Quantitative iodine parameters have been considered a promising tool for distinguishing malignant from benign tumors (12-15). Spectral curves and the effective atomic number $\left(Z_{\text {eff }}\right)$ have helped identify benign and malignant tumors, histological type, and differentiation degree (16-19). Quantitative dualenergy CT (DECT) analysis has distinguished invasive adenocarcinoma from noninvasive or minimally invasive adenocarcinoma among pulmonary ground-glass opacity nodules (13). In addition, the DS-DECT quantitative technique has been used to discriminate the expression status of immunohistochemical biomarkers of invasive breast cancer (20). Previous studies have demonstrated the potential value of quantitative parameters of DECT in the differential diagnosis of benign and malignant thyroid nodules (21-24). However, few studies have used iodine concentration (IC), energy curve, and $Z_{\text {eff }}$ as the quantitative parameters.

With the rapid evolution of machine learning, clustering algorithms can assign data points to discrete groups to maximize within-group similarities and between-group differences. A previous study reported that machine learning of DECT had a better diagnostic performance than qualitative evaluation of CT image features for preoperatively diagnosing cervical lymph node metastases in papillary thyroid cancer patients (25). We hypothesize that the different properties of benign and malignant thyroid nodules can be reflected by the quantitative parameters derived from DS-DECT. The present study obtained quantitative parameters in the nonenhanced and arterial phases and constructed a model to classify benign and malignant thyroid nodules.

We present the following article in accordance with the STARD reporting checklist (available at https://dx.doi. org/10.21037/qims-21-501).

\section{Methods}

\section{Patient collection and thyroid nodule selection}

This was a retrospective study of DS-DECT scans (SOMATOM Drive, Siemens Healthineers, Forchheim, Germany) of the neck region. Data collection was planned after pathological surgical results were obtained. The study was conducted in accordance with the Declaration of Helsinki (as revised in 2013). The study was approved by the Ethics Committee of the Chongqing University Cancer Hospital, and the requirement for informed consent was waived. Patients who underwent CT scanning of thyroid nodules in our hospital between November 2019 and November 2020 were eligible for inclusion. Patients formed a consecutive series. The inclusion criteria were as follows: (I) pathological surgical results were available within a week of the CT examination; (II) the nodules were solid or mixed cystic; (III) only the largest nodule was selected when there were more than two nodules in one lobule. The exclusion criteria were as follows: (I) the presence of obvious artifacts; (II) patients had received a needle biopsy or therapy before the CT examination; (III) the nodule diameter was $<10 \mathrm{~mm}$; (IV) the pathological results were indeterminate. The screening flow chart is shown in Figure 1.

\section{Sample size}

There are no generally accepted approaches to estimate the 


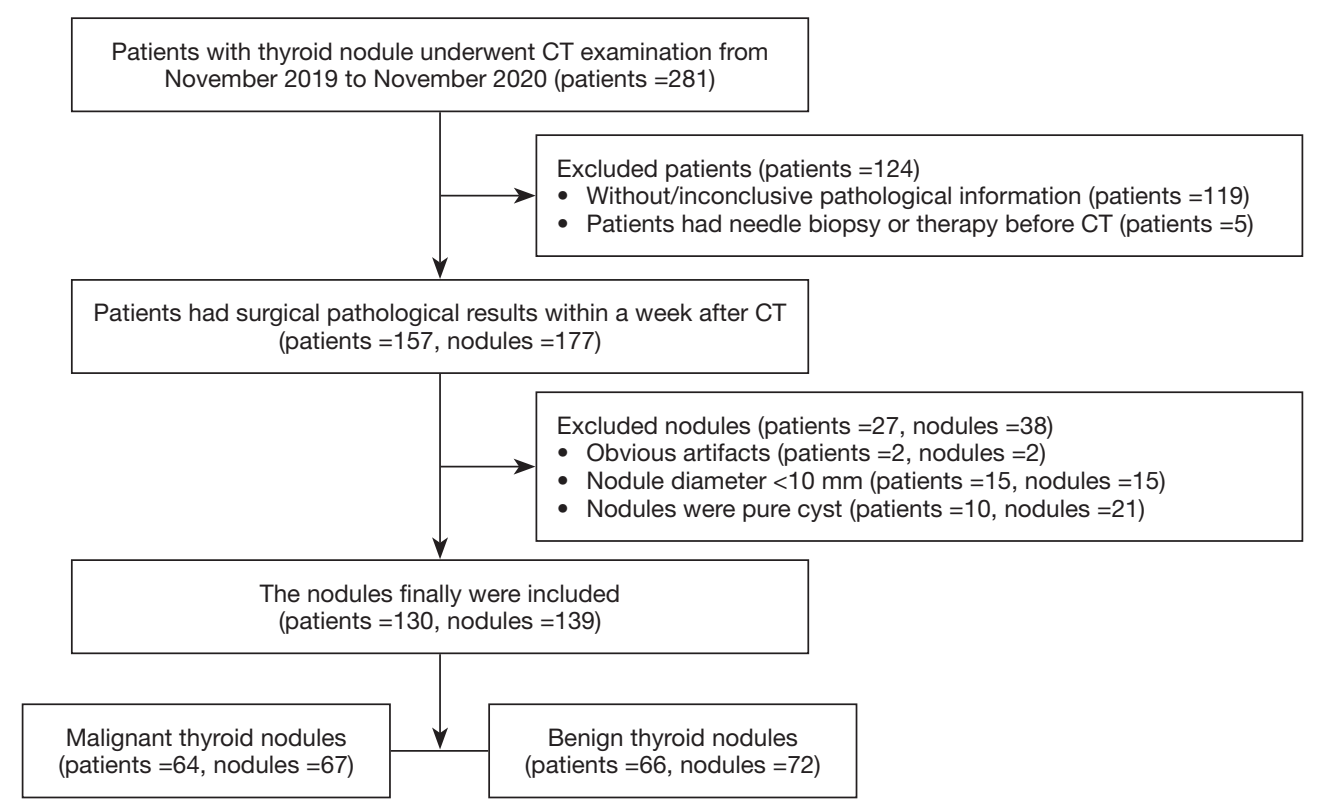

Figure 1 Flowchart showing the screening strategy for patients in this study.

sample size required for derivation and validation studies of prediction models. However, we ensured that the study met the suggested requirements of having at least ten events per candidate variable to derive a model and at least 100 events for the validation study.

\section{Examination method}

A dual-energy protocol was used with tube A (Sn $140 \mathrm{KVp}$, $104 \mathrm{mAs}$ ) and tube B (Sn $100 \mathrm{KVp}, 134 \mathrm{mAs})$ with a reformatted slice thickness of $1.5 \mathrm{~mm}$, a collimation thickness of $32 \times 0.6 \mathrm{~mm}$, a matrix of $512 \times 512$, and a helical pitch of 0.7 . The reconstruction kernel of the monoenergetic image was Q30f, and the iterative reconstruction algorithm used was Advanced Modeled Iterative Reconstruction (ADMIRE). The range extended from the petrous temporal bone to the thoracic entrance. The nonenhanced scan was acquired first. Patients were injected with a nonionic contrast material via an antecubital vein at a rate of $2-2.5 \mathrm{~mL} / \mathrm{s}$ $(1.5 \mathrm{~mL} / \mathrm{kg}$ ioversol, $320 \mathrm{mg} / \mathrm{mL}$ iodine, HENGRUI Medicine, Jiangsu, China). After the injection, $20 \mathrm{~mL}$ of saline was transfused. The arterial phase scans were started using a bolus-tracking technique with a threshold of $100 \mathrm{Hu}$ in the aorta arch and an additional delay of $7 \mathrm{~s}$. The dose length product was $444.29 \pm 156.27 \mathrm{mGy}$. The CT dose index was $19.18 \pm 5.82 \mathrm{mGy}$.

\section{Thyroid nodule analysis}

All dual-energy scanning data were processed in viewer software on a syngo.via workstation (syngo.via VB20A, Dual Energy, Siemens Healthineers). The slice that best showed the boundary and parenchyma of the nodule was selected. The largest possible (round) region of interest (ROI) of the nodule was placed within the enhanced parenchyma of the nodule (Figures 2,3). The measurements were taken by two independent observers [observer 1 (L.L.J) and observer 2 (J.C) with two and six years of experience in thyroid imaging, respectively]. The observers were only aware of the location and diameter of the lesions and were blind to the pathological surgical results. The measurement was repeated one month later by observer 1 .

A quantitative analysis of IC and normalized iodine concentration (NIC) was carried out with virtual nonenhanced module. To minimize variation caused by the patient's hormone and/or circulation status, the NIC was computed according to the IC of the thyroid parenchyma (NIC-P) and carotid artery (NIC-A). The ROI of the carotid artery was placed on the ipsilateral carotid artery. An ROI of the thyroid parenchyma was placed on the normal thyroid parenchyma, as close as possible to the ipsilateral thyroid parenchyma if there was epibiotic normal thyroid parenchyma. To reflect the blood flow of the nodules, the 


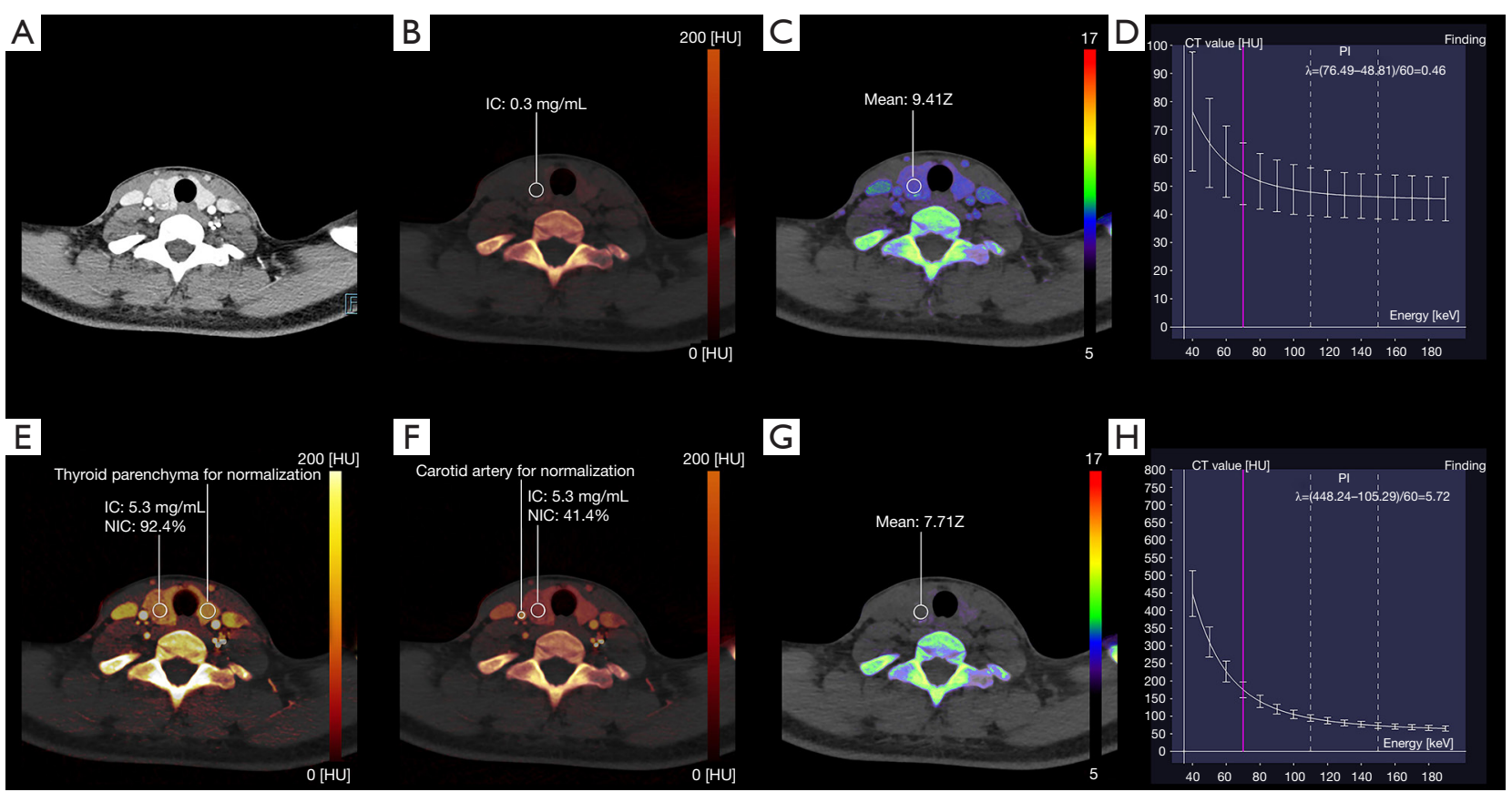

Figure 2 Images of a 23-year-old male with papillary carcinoma: (A) the arterial polyenergetic image is selected as the reference to draw the region of interest; (B) $\mathrm{IC}_{\text {nodule }}$ (nonenhancement) is $0.3 \mathrm{mg} / \mathrm{mL}$; (C) $Z_{\text {eff }}$ (nonenhancement) is 7.71 ; (D) $\lambda_{\mathrm{Hu}}$ (nonenhancement) of the energy curve is 0.46; (E) $\mathrm{IC}_{\text {nodule }}$ (arterial phase) is $5.3 \mathrm{mg} / \mathrm{mL}$, and NIC-P (arterial phase) is $92.4 \%$; (F) $\mathrm{IC}_{\text {nodule }}$ (arterial phase) is $5.3 \mathrm{mg} / \mathrm{mL}$, and NIC-A (arterial phase) is $41.4 \%$; $(\mathrm{G}) \mathrm{Z}_{\text {eff }}$ (arterial phase) is 9.65 ; $(\mathrm{H}) \lambda_{\mathrm{Hu}}$ (arterial phase) of the energy curve is 5.72 . IC, iodine concentration; $Z_{\text {eff }}$, effective atomic number; $\lambda_{\mathrm{Hu}}$, spectral curve slope.

iodine concentration difference (ICD) was computed. The same ROI was applied for IC and NIC measurements in the nonenhanced and arterial phase images. The NIC-P (Eq. [1]), NIC-A (Eq. [2]), and ICD (Eq. [3]) were calculated by the following formulas:

$$
\begin{aligned}
& N I C-P=I C_{\text {nodule }} / I C_{\text {thyroid parenchyma }} \\
& N I C-A=I C_{\text {nodule }} / I C_{\text {carotid artery }} \\
& I C D=I C_{\text {nodule }}(\text { arterial phase })-I C_{\text {nodule }} \text { (nonenhancement) }
\end{aligned}
$$

A quantitative analysis of the slope of the spectral Hounsfield unit curve $\left(\lambda_{\mathrm{Hu}}\right)$ was carried out with monoenergetict in the nonenhanced and arterial phases. Selecting the module as "monoenergetic plus ROI", the largest possible (round) ROI was placed on the thyroid nodule on the monoenergetic images (70 KeV) (26). Lastly, the dual-energy spectral curve was obtained from 40 to $190 \mathrm{keV}$ monochromatic energy images and plotted on the graph as different CT density values for various energy levels. The slope of the spectral Hounsfield unit curve (Eq. [4]) was calculated by the following formula (15):

$$
\lambda_{H \mathrm{u}}=\frac{H u_{40 \mathrm{keV}}-H u_{100 \mathrm{keV}}}{100-40}
$$

Quantitative analysis of $Z_{\text {eff }}$ was carried out with Rho/ $Z$. Rho/Z was measured in the nonenhanced and arterial phases. The same ROI was applied for the $Z_{\text {eff }}$ measurement. Every measurement was acquired on the same slice.

\section{Histopathologic examination}

According to the established convention, the histopathologic examination was used as the reference standard. Tissue samples of each nodule were obtained from the operation. Surgically resected nodules were subjected to an intra-operative frozen section analysis for preliminary risk assessment. The final diagnosis was based on the postoperative paraffin section pathological examination. In the event of suspicious malignant samples or atypical samples, immunohistochemical staining was 

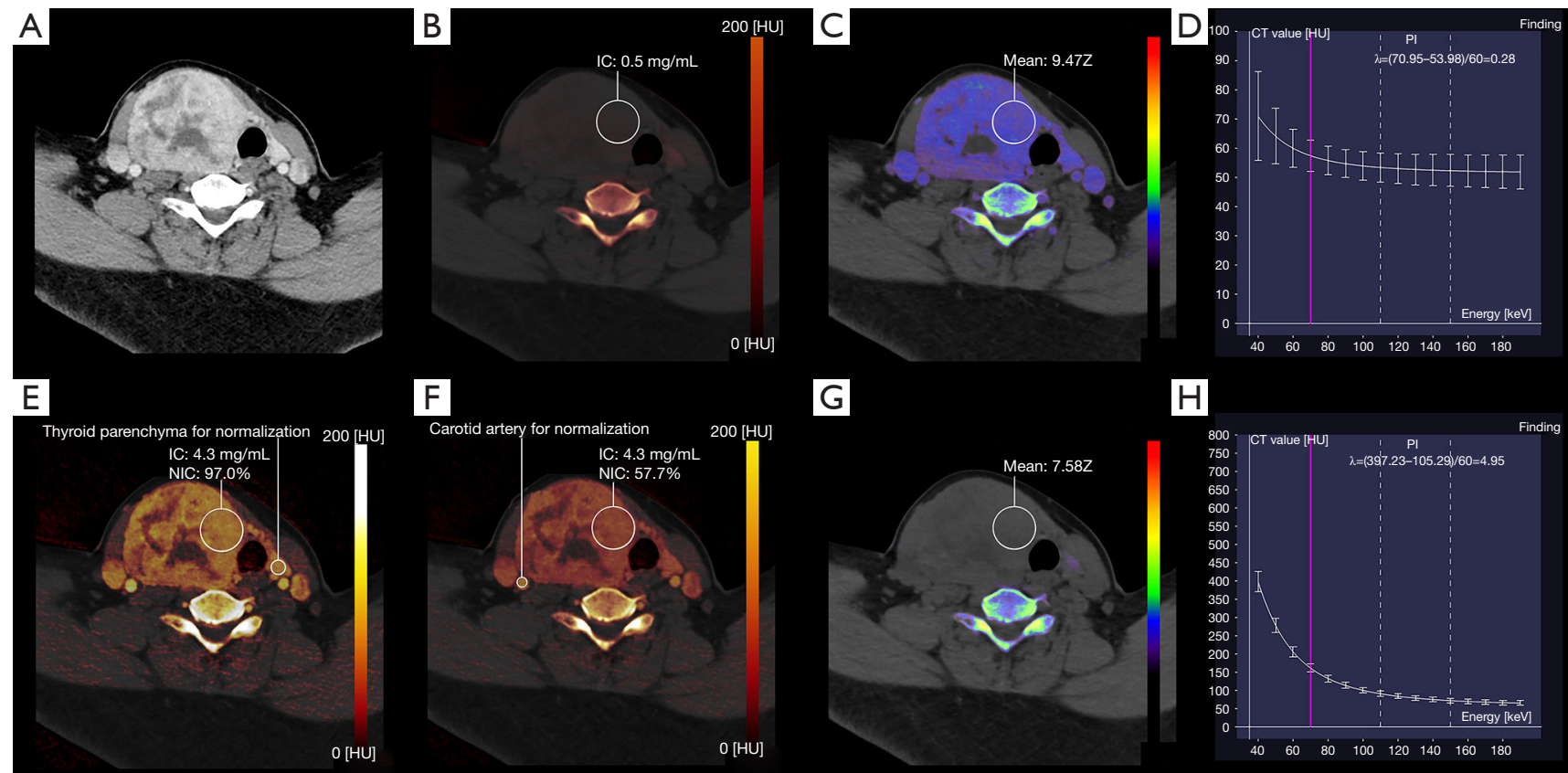

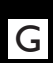

G

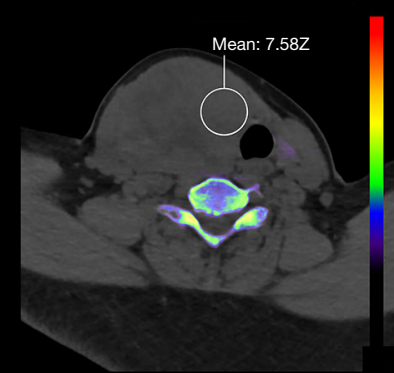

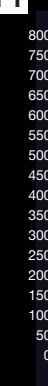

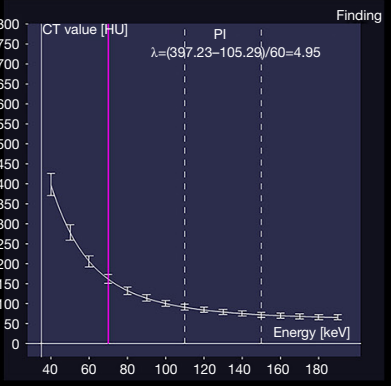

Figure 3 Images of a 41-year-old male with follicular adenoma: (A) the arterial polyenergetic image is selected as the reference to draw the region of interest; (B) $\mathrm{IC}_{\text {nodule }}$ (nonenhancement) is $0.5 \mathrm{mg} / \mathrm{mL}$; (C) $Z_{\text {eff }}$ (nonenhancement) is 7.58 ; (D) $\lambda_{\mathrm{Hu}}$ (nonenhancement) of the energy curve is 0.28 ; (E) $\mathrm{IC}_{\text {nodule }}$ (arterial phase) is $4.3 \mathrm{mg} / \mathrm{mL}$, and NIC-P (arterial phase) is $97.0 \%$; (F) $\mathrm{IC}_{\text {nodule }}$ (arterial phase) is $4.3 \mathrm{mg} / \mathrm{mL}$, and NIC-A (arterial phase) is $57.7 \%$; $(\mathrm{G}) \mathrm{Z}_{\text {eff }}$ (arterial phase) is $9.39 ;(\mathrm{H}) \lambda_{\mathrm{Hu}}$ (arterial phase) of the energy curve is 4.95 . IC, iodine concentration; $Z_{\text {eff }}$, effective atomic number; $\lambda_{\mathrm{Hu}}$, spectral curve slope.

applied to differentiate benign from malignant nodules. An experienced pathologist determined all diagnoses. According to the pathological results, the nodules were assigned to either the benign or malignant groups.

\section{Statistical analysis}

Statistical analyses were performed using SPSS (version 25.0), and the results were considered statistically significant at $\mathrm{P}<0.05$. All variables of the quantitative parameters derived from multiple phase DS-DECT were exploratory. The interclass correlation coefficient (ICC) was used to test the interobserver reliability. Multiple quantitative parameters derived from DS-DECT were compared between the benign group and the malignant group. Tests for normality were performed using the ShapiroWilk test. Normally distributed continuous variables were compared using Student's $t$-test, which included NIC-P (arterial phase), $\lambda_{\mathrm{Hu}}$ (nonenhancement), and $\mathrm{Z}_{\text {eff }}$ (arterial phase). Other variables were compared using the MannWhitney $\mathrm{U}$ test, which included $\mathrm{IC}_{\text {nodule }}$ (nonenhancement),
$\mathrm{IC}_{\text {nodule }}$ (arterial phase), NIC-A (arterial phase), ICD, $\lambda_{\mathrm{Hu}}$ (arterial phase), and $Z_{\text {eff }}$ (nonenhancement). If the $\mathrm{P}$ values for the variables were $<0.05$, the quantitative parameters derived from the multiple phase DS-DECT were entered into a binary logistic regression analysis to calculate the odds ratios (OR) and 95\% confidence intervals (CI). All statistically significant variables were entered into a further multiple logistic regression analysis to build a prediction model of malignant nodules.

Machine learning was performed using Python (version 3.7) and R (version 4.0.1). Python was used to establish the feature selection and to construct a prediction model. R was used to compare the diagnostic efficacy. The patients were randomly assigned to the training group and testing group at a ratio of $2: 1$. The training group was used for feature selection and to create the prediction model. We used the least absolute shrinkage and selection operator (LASSO) algorithm to select key features. The LASSO algorithm was used to eliminate features with the greatest redundancy. A naive LASSO penalty was used to optimize the classifier. A linear discriminant analysis (LDA) based on the key 
Table 1 Quantification parameters of DS-DECT between the benign and malignant groups

\begin{tabular}{|c|c|c|c|}
\hline Parameters & Malignant group $(n=67)$ & Benign group $(n=72)$ & $\mathrm{P}$ \\
\hline $\mathrm{IC}_{\text {nodule }}$ (arterial phase) $(\mathrm{mg} / \mathrm{mL})$ & $3.21 \pm 1.46$ & $2.43 \pm 1.56$ & 0.001 \\
\hline NIC-P (arterial phase) (\%) & $68.93 \pm 29.19$ & $51.84 \pm 31.22$ & 0.002 \\
\hline NIC-A (arterial phase) (\%) & $33.63 \pm 15.65$ & $30.52 \pm 20.44$ & 0.062 \\
\hline$\lambda_{\mathrm{Hu}}$ (nonenhancement) & $2.19 \pm 1.93$ & $1.61 \pm 1.96$ & 0.007 \\
\hline$\lambda_{\text {Нu }}$ (arterial phase) & $3.28 \pm 1.58$ & $2.32 \pm 1.72$ & 0.001 \\
\hline$Z_{\text {eff }}$ (nonenhancement) & $7.80 \pm 0.22$ & $7.62 \pm 0.26$ & $<0.001$ \\
\hline $\mathrm{Z}_{\text {eff }}$ (arterial phase) & $8.86 \pm 0.62$ & $8.61 \pm 0.72$ & 0.039 \\
\hline
\end{tabular}

DS-DECT, dual-source dual-energy computed tomography; IC, iodine concentration; NIC-P, IC $\mathrm{C}_{\text {nodule }} / \mathrm{IC}_{\text {thyroid parenchyma }} ; \mathrm{NIC}-\mathrm{A}$, IC $\mathrm{nodule}_{\mathrm{I}} / \mathrm{IC}_{\text {carotid artery }}$; $I C D, I C_{\text {nodule_arterial phase }}-I_{\text {nodule_nonenhancement }} ; \lambda_{\text {Hu }}$, slope of the spectral Hounsfield unit curve; $Z_{\text {eff }}$, effective atomic number.

features was used to construct the models. Before the model construction, data normalization was performed to avoid bigger ranges dominating those in smaller ranges. We used 5 -fold cross-validation results to evaluate the performance of a specific machine learning classifier, where "accuracy" was chosen as the optimization metric to select the classifier. The key features included $Z_{\text {eff }}$ (nonenhancement), $\lambda_{\mathrm{Hu}}$ (arterial phase), ICD, NIC-P (arterial phase), and IC $_{\text {nodule }}$ (arterial phase). The DeLong test was used to compare the diagnostic efficacy.

\section{Results}

\section{Clinical data}

A total of 281 patients were identified for this study: 64 males and 217 females, with an age range of 16-86 years. Of these, 117 patients did not show pathological results, and the clinician diagnosed their nodules as benign. The pathological results of the two patients were inconclusive. One case had follicular adenoma with atypical hyperplasia, and another case had nodular goiters with focal cancerous lesions. Five patients with papillary carcinoma had received a needle biopsy or therapy before the CT examination. The images from two patients had obvious artifacts. Fifteen patients had micropapillary carcinoma, and ten patients had follicular adenoma with cystic degeneration. Ultimately, 130 patients were included in this study, of which 102 were females, and 28 were males, with an age range of $18-84$ years. The malignant group comprised 52 females and 12 males aged 18-84 years old. The benign group comprised 50 females and 16 males aged 26-73 years old. Among the 139 nodules from 130 patients, 67 nodules were malignant (64 papillary carcinomas, two follicular carcinomas, and one medullary carcinoma), and 72 nodules were benign (46 follicular adenomas, 20 nodular goiters, two subacute thyroiditis, two Hashimoto's thyroiditis, one granulomatous thyroiditis, and one oxyphilic cell adenoma). There were no adverse events in this study.

\section{Comparison of the DS-DECT quantitative parameters between the malignant and benign groups}

The results are summarized in Table 1. The malignant group had a significantly higher $\mathrm{IC}_{\text {nodule }}$ (arterial phase) ( $\mathrm{P}=0.001)$, NIC-P (arterial phase) $(\mathrm{P}=0.002)$, ICD $(\mathrm{P}<0.001)$, $\lambda_{\mathrm{Hu}}$ (nonenhancement) $(\mathrm{P}=0.007), \lambda_{\mathrm{Hu}}$ (arterial phase) $(\mathrm{P}=0.001), Z_{\text {eff }}$ (nonenhancement) $(\mathrm{P}<0.001)$ and $Z_{\text {eff }}$ (arterial phase) $(\mathrm{P}=0.039)$ than the benign group (Table 1). The interobserver reproducibility for measuring thyroid nodules with the DS-DECT parameters is summarized in Table 2.

\section{Binary logistic regression analysis of DS-DECT quantitative parameters for the prediction of malignant nodules}

In the univariate binary logistic regression analysis, $\mathrm{IC}_{\text {nodule }}$ (arterial phase) $(\mathrm{P}=0.004), \mathrm{NIC}-\mathrm{P}$ (arterial phase) $(\mathrm{P}=0.003), \operatorname{ICD}(\mathrm{P}=0.001), \lambda_{\mathrm{Hu}}$ (arterial phase) $(\mathrm{P}=0.001)$, $Z_{\text {eff }}$ (nonenhancement) $(\mathrm{P}<0.001)$, and $Z_{\text {eff }}$ (arterial phase) $(\mathrm{P}=0.042)$ reached statistical significance (Table 3). All the 
Table 2 The interobserver and intraobserver reproducibility for measuring thyroid nodules with DS-DECT parameters

\begin{tabular}{lcc}
\hline Parameters & Interobserver $(95 \% \mathrm{Cl})$ & Intraobserver $(95 \% \mathrm{Cl})$ \\
\hline $\mathrm{IC}_{\text {nodule }}$ (nonenhancement) & $0.857(0.805-0.895)$ & $0.925(0.897-0.946)$ \\
$\mathrm{IC}_{\text {nodule }}$ (arterial phase) & $0.951(0.932-0.965)$ & $0.986(0.980-0.990)$ \\
$\mathrm{NIC}-\mathrm{P}$ (arterial phase) & $0.962(0.947-0.973)$ & $0.973(0.963-0.981)$ \\
$\mathrm{NIC}-\mathrm{A}$ (arterial phase) & $0.889(0.849-0.920)$ & $0.937(0.913-0.955)$ \\
$\lambda_{\text {Hu }}$ (nonenhancement) & $0.979(0.971-0.985)$ & $0.995(0.993-0.996)$ \\
$\lambda_{\text {Hu }}$ (arterial phase) & $0.893(0.853-0.922)$ & $0.971(0.959-0.979)$ \\
$Z_{\text {eff }}$ (nonenhancement) & $0.626(0.513-0.717)$ & $0.838(0.781-0.882)$ \\
$Z_{\text {eff }}$ (arterial phase) & $0.832(0.772-0.877)$ & $0.950(0.931-0.964)$ \\
\hline
\end{tabular}

DS-DECT, dual-source dual-energy computed tomography; Cl, confidence interval; ROI, region of interest; IC, iodine concentration; NIC-P, $I \mathrm{I}_{\text {nodule }} / \mathrm{IC}_{\text {thyroid parenchyma }} ; \mathrm{NIC}-\mathrm{A}, \mathrm{IC}_{\text {nodule }} / \mathrm{IC}_{\text {carotid artery }} ; \lambda_{\text {Hu}}$, slope of the spectral Hounsfield unit curve; $\mathrm{Z}_{\text {eff }}$, effective atomic number.

Table 3 The univariate binary logistic regression analysis with DS-DECT parameters for prediction of malignant nodules

\begin{tabular}{|c|c|c|c|c|}
\hline Parameters & $95 \% \mathrm{Cl}$ & OR & $\beta$ & $\mathrm{P}$ \\
\hline NIC-P (arterial phase) & $1.006-1.030$ & 8.067 & 1.018 & 0.003 \\
\hline ICD & $1.228-2.215$ & 11.067 & 1.649 & 0.001 \\
\hline$\lambda_{\mathrm{Hu}}$ (nonenhancement) & $0.977-1.391$ & 2.895 & 1.166 & 0.089 \\
\hline$Z_{\text {eff }}$ (nonenhancement) & $4.318-13.808$ & 13.067 & 24.418 & $<0.001$ \\
\hline$Z_{\text {eff }}$ (arterial phase) & $1.019-2.827$ & 4.135 & 1.697 & 0.042 \\
\hline
\end{tabular}

DS-DECT, dual-source dual-energy computed tomography; OR, odds ratio; $\beta$, partial regression coefficient; $\mathrm{Cl}$, confidence interval; IC, iodine concentration; NIC-P, IC $C_{\text {nodule }} / I_{\text {thyroid parenchyma; }} I C D$, IC $C_{\text {nodule_arterial phase }}-I C_{\text {nodule_nonenhancement }} ; \lambda_{H u}$, slope of the spectral Hounsfield unit curve; $Z_{\text {eff, }}$ effective atomic number.

Table 4 The predictive performance of the binary logistic regression analysis model

\begin{tabular}{llll}
\hline Sample & Sensitivity $(95 \% \mathrm{Cl})$ & Specificity $(95 \% \mathrm{Cl})$ & Likelihood ratio \\
\hline Total sample $(\mathrm{n}=139)$ & $0.821(0.708-0.904)$ & $0.667(0.546-0.773)$ & 2.463 \\
\hline
\end{tabular}

$\mathrm{Cl}$, confidence interval.

statistically significant variables were entered into a further multiple logistic regression analysis to build the prediction model for malignant nodules. The prediction model had an AUC of 0.76 , sensitivity of 0.821 , and specificity of 0.667 (Table 4). The ROCs of the prediction are shown in Figure 4.

\section{Machine learning result of DS-DECT quantitative parameters for the prediction of malignant nodules}

There were 45 malignant nodules and 48 benign nodules in the training cohort. There were 22 malignant nodules and 24 benign nodules in the testing cohort. The 5 -fold crossvalidation curves of the model training procedure are shown in Figure 5. In the training cohort, the prediction model had an AUC of 0.86 , sensitivity of 0.822 , specificity of 0.791 , and accuracy of 0.806 (Table 5). In the testing cohort, the prediction model had an AUC of 0.84 , a sensitivity of 0.727 , a specificity of 0.750 , and an accuracy of 0.739 (Table 5). The ROCs of the training and testing groups are shown in Figure 6. The DeLong test showed that the AUC of the 


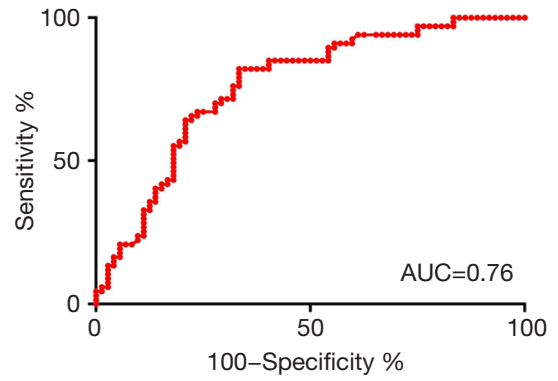

Figure 4 The receiver operating characteristic curve of the binary logistic regression analysis model shows that the area under the curve is 0.76 .

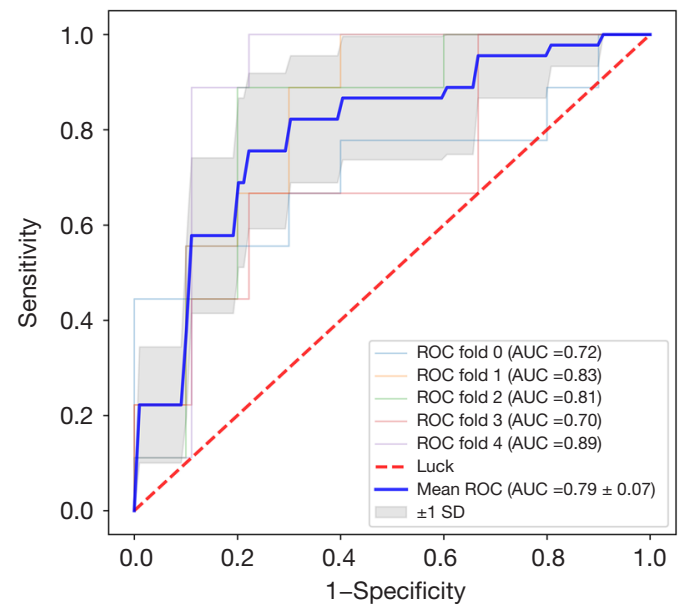

Figure 5 The 5 -fold cross-validation curves of the model training procedure. machine learning was statistically higher than the logistic regression analysis (Table 6).

\section{Discussion}

In recent years, an increasing number of studies have reported that DS-DECT can be used to diagnose malignant and benign tumors $(22,27-29)$. The present study showed that quantitative parameters derived from DS-DECT could differentiate benign from malignant thyroid nodules.

The thyroid is the only human organ rich in iodine, so measuring the iodine concentration can reflect thyroid

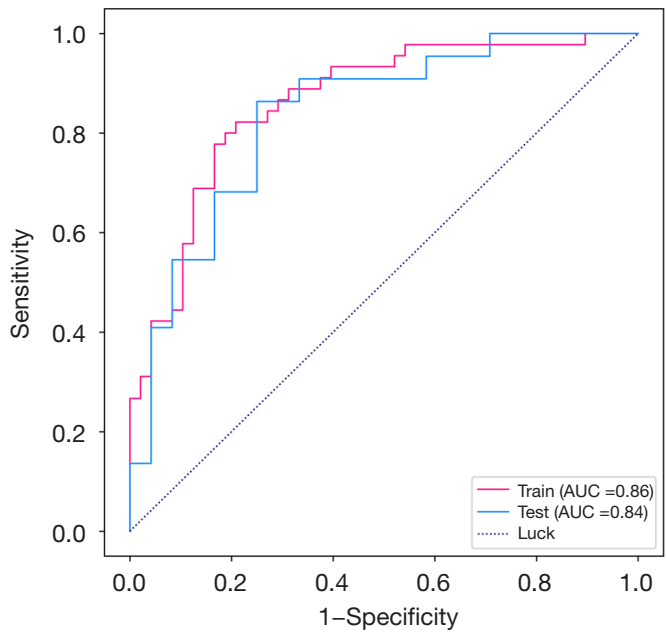

Figure 6 The receiver operating characteristic curve of the machine learning model shows that the area under the curve in the training group is 0.86 , and the area under the curve in the test group is 0.84 .

Table 5 The predictive performance of the machine learning model

\begin{tabular}{lccccc}
\hline Cohort & Sensitivity & Specificity & Accuracy & Positive predictive value & Negative predictive value \\
\hline Training cohort $(n=93)$ & 0.822 & 0.791 & 0.806 & 0.787 & 0.826 \\
Testing cohort $(n=46)$ & 0.727 & 0.750 & 0.739 & 0.727 & 0.750 \\
\hline
\end{tabular}

Table 6 The result of the DeLong test between machine learning and logistic regression analysis models

\begin{tabular}{ccc}
\hline & Training cohort $(n=93)$ & Testing cohort $(n=46)$ \\
\hline$P$ & 0.029 & 0.036 \\
\hline
\end{tabular}


function to some extent. A previous study verified that ${ }^{123}$ I uptake at 3 hours was negatively correlated with IC evaluated by DECT (30). In this study, the $\mathrm{IC}_{\text {nodule }}$ (nonenhancement) showed no statistical significance between the malignant and benign groups, which contrasts to a previous study (31). In theory, the $\mathrm{IC}_{\text {nodule }}$ (nonenhancement) should be lower in the malignant group than in the benign group because thyroid follicular cells have an iodine uptake function that barely exists in malignant lesions but is present in benign lesions, such as adenoma, nodular goiter, and subacute thyroiditis (32-34). However, the Siemens Healthineers' dual-energy model was designed for liver imaging and cannot identify microcalcification. Therefore, microcalcification of papillary carcinoma could affect the measurement of IC. Our study used the IC nodule (arterial phase), NIC-P (arterial phase), and NIC-A (arterial phase), which are mainly influenced by contrast material. Our results suggested that the IC nodule (arterial phase) and NIC-P (arterial phase) were significantly higher in the malignant group than in the benign group. A previous study reported that the AUC of NIC was better than IC (21), which was partly similar to this study.

Our results suggested that $\lambda_{\mathrm{Hu}}$ was higher in the malignant group than in the benign group. The dual-energy spectral curve was obtained from various monochromatic energy images and plotted on the graph as different CT density values concerning various energy levels. The spectral curve may be used to differentiate fibrous, fat, calcium, and iodine because each of these has different attenuations at different energy levels. A previous study reported that $\lambda_{\mathrm{Hu}}$ in papillary carcinoma was described as negative, which could be explained by the presence of lipid materials in the cells (23). However, no numerical values or statistical results were shown in this previous study. In fact, the $\lambda_{\mathrm{Hu}}$ in some patients with follicular adenoma, nodular goiters, subacute thyroiditis, and granulomatous thyroiditis was also negative in our study. Therefore, we infer that lipid materials not only exist in papillary carcinoma but also exist in other non-cancerous lesions. Previous studies have suggested that thyroid-stimulating hormones can induce thyroid lipid metabolism disorder $(35,36)$.

$Z_{\text {eff }}$ reflected the atomic number of the compound material; the denser the compound, the higher the effective atomic number $(37,38)$. In lung cancer, gastric cancer, and rectal cancer studies, $Z_{\text {eff }}$ has proved helpful in the preoperative identification of benign and malignant tumors (39-41). Our study found that $Z_{\text {eff }}$ (nonenhancement) and $Z_{\text {eff }}$ (arterial phase) was significantly higher in the malignant group than in the benign group. In addition, $Z_{\text {eff }}$ (nonenhancement) was the most important indicator for predicting malignant thyroid nodules. Malignant nodules are characterized by a higher cell density than benign nodules (42); therefore, malignant nodules may have a higher $Z_{\text {eff }}$ than benign nodules. In addition, microcalcification may result in a higher $Z_{\text {eff }}$ in the malignant group than in the benign group. In the arterial phase, a higher $Z_{\text {eff }}$ may also be related to higher perfusion in the malignant group than in the benign group (43).

In this study, machine learning showed a better diagnostic performance than binary logistic regression analysis because the LDA variable was calculated for each case through singular value decomposition, minimizing the intraclass variance and maximizing the interclass variance (44). In addition, as a commonly used data process method, LDA has a simple principle and a short calculation time. The results of our study indicated that "Lasso LDA" improved both the AUC and specificity in the prediction model of malignant thyroid nodules, compared to the binary logistic regression analysis. This is because "Lasso LDA" adopts a naive Lasso penalty in LDA without taking the matrix structure into account, and the logistic regression was regularized (45).

This study does have some limitations. First, the ROI included a slice rather than the entire nodule. Whether the parameters acquired from the entire nodule are better than those obtained from a slice is an issue that needs further study. Second, we adopted the monoenergetic images $(70 \mathrm{keV})$ with the clearest boundaries to obtain a highly accurate ROI when necessary. The different monoenergetic image qualities for showing lesions at each $\mathrm{keV}$ level should be explored. Third, selection bias is inevitable because this is a retrospective study. For instance, this result did not apply to nodule diameters $<10 \mathrm{~mm}$, because the repeatability was poor owing to the volume effect. Lastly, the results are limited to the screening criteria and the DSDECT scanning protocol.

In conclusion, this study suggests that multiple quantitative parameters of DS-DECT may serve as imaging markers to differentiate between benign and malignant nodules in the thyroid. The machine learning model constructed with these parameters exhibited excellent performance for the differential diagnosis.

\section{Acknowledgments}

The authors thank all volunteers who participated in 
the study and the staff of the Department of Radiology, Chongqing University Cancer Hospital, China, for their selfless and valuable assistance. We acknowledge the support of Xiaoyue Zhang from Siemens scientific research.

Funding: This study received funding from the National Natural Science Foundation of China (Grant No. 82071883), the Combination Projects of Medicine and Engineering of the Fundamental Research Funds for the Central Universities in 2019 (Project No. 2019CDYGYB008), the Chongqing Key Medical Research Project of Combination of Science and Medicine (Grant No. 2019ZDXM007), and the 2019 SKY Imaging Research Fund of the Chinese International Medical Foundation (Project No. Z-2014-07-1912-10).

\section{Footnote}

Reporting Checklist: The authors have completed the STARD reporting checklist. Available at https://dx.doi. org/10.21037/qims-21-501

Conflicts of Interest: All authors have completed the ICMJE uniform disclosure form (available at https:// dx.doi.org/10.21037/qims-21-501). JZ receives fundings from the National Natural Science Foundation of China (Grant No. 82071883), combination projects of medicine and engineering of the Fundamental Research Funds for the Central Universities in 2019 (project No. 2019CDYGYB008), the Chongqing key medical research project of combination of science and medicine (grant No. 2019ZDXM007), and the 2019 SKY Imaging Research Fund of the Chinese International Medical Foundation (project No. Z-2014-07-1912-10). The other authors have no conflicts of interest to declare.

Ethical Statement: The authors are accountable for all aspects of the work in ensuring that questions related to the accuracy or integrity of any part of the work are appropriately investigated and resolved. The study was conducted in accordance with the Declaration of Helsinki (as revised in 2013). This retrospective study was approved by the Review Committee of Chongqing University Cancer Hospital, and informed consent from the study participants was waived.

Open Access Statement: This is an Open Access article distributed in accordance with the Creative Commons Attribution-NonCommercial-NoDerivs 4.0 International
License (CC BY-NC-ND 4.0), which permits the noncommercial replication and distribution of the article with the strict proviso that no changes or edits are made and the original work is properly cited (including links to both the formal publication through the relevant DOI and the license). See: https://creativecommons.org/licenses/by-nc-nd/4.0/.

\section{References}

1. Nguyen XV, Choudhury KR, Eastwood JD, Lyman GH, Esclamado RM, Werner JD, Hoang JK. Incidental thyroid nodules on CT: evaluation of 2 risk-categorization methods for work-up of nodules. AJNR Am J Neuroradiol 2013;34:1812-7.

2. Ahmed S, Horton KM, Jeffrey RB Jr, Sheth S, Fishman EK. Incidental thyroid nodules on chest CT: Review of the literature and management suggestions. AJR Am J Roentgenol 2010;195:1066-71.

3. Durante C, Grani G, Lamartina L, Filetti S, Mandel SJ, Cooper DS. The Diagnosis and Management of Thyroid Nodules: A Review. JAMA 2018;319:914-24.

4. Richman DM, Benson CB, Doubilet PM, Wassner AJ, Asch E, Cherella CE, Smith JR, Frates MC. Assessment of American College of Radiology Thyroid Imaging Reporting and Data System (TI-RADS) for Pediatric Thyroid Nodules. Radiology 2020;294:415-20.

5. Tappouni RR, Itri JN, McQueen TS, Lalwani N, Ou JJ. ACR TI-RADS: Pitfalls, Solutions, and Future Directions. Radiographics 2019;39:2040-52.

6. Song M, Yue Y, Jin Y, Guo J, Zuo L, Peng H, Chan Q. Intravoxel incoherent motion and ADC measurements for differentiating benign from malignant thyroid nodules: utilizing the most repeatable region of interest delineation at 3.0 T. Cancer Imaging 2020;20:9.

7. Montgomery J, Hendry J, Van der Horst C, Hunter MA, MacKenzie K, Hilmi O. Cytological accuracy and radiological staging in patients with thyroid cancer in Glasgow. Eur Arch Otorhinolaryngol 2016;273:2741-6.

8. Goo HW, Goo JM. Dual-Energy CT: New Horizon in Medical Imaging. Korean J Radiol 2017;18:555-69.

9. Yoon JH, Chang W, Lee ES, Lee SM, Lee JM. Double Low-Dose Dual-Energy Liver CT in Patients at HighRisk of HCC: A Prospective, Randomized, Single-Center Study. Invest Radiol 2020;55:340-8.

10. Hickethier T, Kroeger JR, Lennartz S, Doerner J, Maintz $\mathrm{D}$, Chang DH. Venous-phase chest CT with reduced contrast medium dose: Utilization of spectral low keV monoenergetic images improves image quality. Eur J 
Radiol 2020;122:108756.

11. Peng Y, Ye J, Liu C, Jia H, Sun J, Ling J, Prince M, Li C, Luo $\mathrm{X}$. Simultaneous hepatic iron and fat quantification with dual-energy CT in a rabbit model of coexisting iron and fat. Quant Imaging Med Surg 2021;11:2001-12.

12. Chang S, Hur J, Im DJ, Suh YJ, Hong YJ, Lee HJ, Kim YJ, Choi BW. Dual-energy CT-based iodine quantification for differentiating pulmonary artery sarcoma from pulmonary thromboembolism: a pilot study. Eur Radiol 2016;26:3162-70.

13. Son JY, Lee HY, Kim JH, Han J, Jeong JY, Lee KS, Kwon OJ, Shim YM. Quantitative CT analysis of pulmonary ground-glass opacity nodules for distinguishing invasive adenocarcinoma from non-invasive or minimally invasive adenocarcinoma: the added value of using iodine mapping. Eur Radiol 2016;26:43-54.

14. Benveniste AP, de Castro Faria S, Broering G, Ganeshan DM, Tamm EP, Iyer RB, Bhosale P. Potential Application of Dual-Energy CT in Gynecologic Cancer: Initial Experience. AJR Am J Roentgenol 2017;208:695-705.

15. Li M, Zheng X, Gao F, Xiao L, Hua Y. Spectral CT imaging of intranodular hemorrhage in cases with challenging benign thyroid nodules. Radiol Med 2016;121:279-90.

16. Volterrani L, Gentili F, Fausto A, Pelini V, Megha T, Sardanelli F, Mazzei MA. Dual-Energy CT for Locoregional Staging of Breast Cancer: Preliminary Results. AJR Am J Roentgenol 2020;214:707-14.

17. Patel BN, Boltyenkov AT, Martinez MG, Mastrodicasa D, Marin D, Jeffrey RB, Chung B, Pandharipande P, Kambadakone A. Cost-effectiveness of dual-energy CT versus multiphasic single-energy CT and MRI for characterization of incidental indeterminate renal lesions. Abdom Radiol (NY) 2020;45:1896-906.

18. Karino T, Ohira S, Kanayama N, Wada K, Ikawa T, Nitta Y, Washio H, Miyazaki M, Teshima T. Determination of optimal virtual monochromatic energy level for target delineation of brain metastases in radiosurgery using dualenergy CT. Br J Radiol 2020;93:20180850.

19. Wang X, Liu D, Zeng X, Jiang S, Li L, Yu T, Zhang J. Dual-energy CT quantitative parameters for the differentiation of benign from malignant lesions and the prediction of histopathological and molecular subtypes in breast cancer. Quant Imaging Med Surg 2021;11:1946-57.

20. Wang X, Liu D, Zeng X, Jiang S, Li L, Yu T, Zhang J. Dual-energy CT quantitative parameters for evaluating Immunohistochemical biomarkers of invasive breast cancer. Cancer Imaging 2021;21:4.
21. Li HW, Wu XW, Liu B, Liu WD, Gao N. Clinical values of of gemstone spectral CT in diagnosing thyroid disease. J Xray Sci Technol 2015;23:45-56.

22. Li L, Wang Y, Luo DH, Zhao YF, Lin M, Guo W, Hu L, Zhou CW, Zhao XM. Diagnostic value of singlesource dual-energy spectral computed tomography for papillary thyroid microcarcinomas. J Xray Sci Technol 2017;25:793-802.

23. Li M, Zheng X, Li J, Yang Y, Lu C, Xu H, Yu B, Xiao L, Zhang G, Hua Y. Dual-energy computed tomography imaging of thyroid nodule specimens: comparison with pathologic findings. Invest Radiol 2012;47:58-64.

24. Liu X, Ouyang D, Li H, Zhang R, Lv Y, Yang A, Xie C. Papillary thyroid cancer: dual-energy spectral CT quantitative parameters for preoperative diagnosis of metastasis to the cervical lymph nodes. Radiology 2015;275:167-76.

25. Zhou Y, Su GY, Hu H, Ge YQ, Si Y, Shen MP, Xu XQ, Wu FY. Radiomics analysis of dual-energy CT-derived iodine maps for diagnosing metastatic cervical lymph nodes in patients with papillary thyroid cancer. Eur Radiol 2020;30:6251-62.

26. Lam S, Gupta R, Levental M, Yu E, Curtin HD, Forghani R. Optimal Virtual Monochromatic Images for Evaluation of Normal Tissues and Head and Neck Cancer Using Dual-Energy CT. AJNR Am J Neuroradiol 2015;36:1518-24.

27. Zhang D, Xu A. Application of dual-source CT perfusion imaging and MRI for the diagnosis of primary liver cancer. Oncol Lett 2017;14:5753-8.

28. Wang M, Li B, Sun H, Huang T, Zhang X, Jin K, Wang F, Luo X. Correlation study between dual source CT perfusion imaging and the microvascular composition of solitary pulmonary nodules. Lung Cancer 2019;130:115-20.

29. Özdeniz İ, İdilman İS, Köklü S, Hamaloğlu E, Özmen M, Akata D, Karçaaltıncaba M. Dual-energy CT characteristics of colon and rectal cancer allows differentiation from stool by dual-source CT. Diagn Interv Radiol 2017;23:251-6.

30. Binh DD, Nakajima T, Otake H, Higuchi T, Tsushima Y. Iodine concentration calculated by dual-energy computed tomography (DECT) as a functional parameter to evaluate thyroid metabolism in patients with hyperthyroidism. BMC Med Imaging 2017;17:43.

31. Gao SY, Zhang XY, Wei W, Li XT, Li YL, Xu M, Sun YS, Zhang XP. Identification of benign and malignant thyroid nodules by in vivo iodine concentration measurement using 
single-source dual energy CT: A retrospective diagnostic accuracy study. Medicine (Baltimore) 2016;95:e4816.

32. Portulano C, Paroder-Belenitsky M, Carrasco N. The $\mathrm{Na}+/ \mathrm{I}-$ symporter (NIS): mechanism and medical impact. Endocr Rev 2014;35:106-49.

33. Kogai T, Hershman JM, Motomura K, Endo T, Onaya T, Brent GA. Differential regulation of the human sodium/iodide symporter gene promoter in papillary thyroid carcinoma cell lines and normal thyroid cells. Endocrinology 2001;142:3369-79.

34. Liou MJ, Lin JD, Chan EC, Liu FH, Chao TC, Weng HF. Detection of mRNA of sodium iodide symporter in benign and malignant human thyroid tissues. Cancer Lett 2000;160:75-80.

35. Dai Z, Yan H, Wang K, Zhong S, Zhou M, Zheng D, Zhu H. MicroRNA-22 regulates thyroid cell growth and lipid accumulation via IL6R. Front Biosci (Landmark Ed) 2019;24:1350-62.

36. Wang F, Tan Y, Wang C, Zhang X, Zhao Y, Song X, Zhang B, Guan Q, Xu J, Zhang J, Zhang D, Lin H, Yu C, Zhao J. Thyroid-stimulating hormone levels within the reference range are associated with serum lipid profiles independent of thyroid hormones. J Clin Endocrinol Metab 2012;97:2724-31.

37. Mileto A, Allen BC, Pietryga JA, Farjat AE, Zarzour JG, Bellini D, Ebner L, Morgan DE. Characterization of Incidental Renal Mass With Dual-Energy CT: Diagnostic Accuracy of Effective Atomic Number Maps for Discriminating Nonenhancing Cysts From Enhancing Masses. AJR Am J Roentgenol 2017;209:W221-30.

38. Garcia LI, Azorin JF, Almansa JF. A new method

Cite this article as: Jiang L, Liu D, Long L, Chen J, Lan X, Zhang J. Dual-source dual-energy computed tomographyderived quantitative parameters combined with machine learning for the differential diagnosis of benign and malignant thyroid nodules. Quant Imaging Med Surg 2022;12(2):967-978. doi: 10.21037/qims-21-501 to measure electron density and effective atomic number using dual-energy CT images. Phys Med Biol 2016;61:265-79.

39. Yang F, Dong J, Wang X, Fu X, Zhang T. Non-small cell lung cancer: Spectral computed tomography quantitative parameters for preoperative diagnosis of metastatic lymph nodes. Eur J Radiol 2017;89:129-35.

40. Böning G, Feldhaus F, Adelt S, Kahn J, Fehrenbach $\mathrm{U}$, Streitparth F. Clinical routine use of virtual monochromatic datasets based on spectral CT in patients with hypervascularized abdominal tumors-evaluation of effectiveness and efficiency. Acta Radiol 2019;60:425-32.

41. Al-Najami I, Mahmoud Sheta H, Baatrup G. Differentiation between malignant and benign rectal tumors by dual-energy computed tomography - a feasibility study. Acta Oncol 2019;58:S55-9.

42. Shi RY, Yao QY, Zhou QY, Lu Q, Suo ST, Chen J, Zheng WJ, Dai YM, Wu LM, Xu JR. Preliminary study of diffusion kurtosis imaging in thyroid nodules and its histopathologic correlation. Eur Radiol 2017;27:4710-20.

43. Wendl CM, Janke M, Jung W, Stroszczysnski C, Jung EM. Contrast-enhanced ultrasound with perfusion analysis for the identification of malignant and benign tumours of the thyroid gland. Clin Hemorheol Microcirc 2015;63:113-21.

44. Weyers BW, Marsden M, Sun T, Bec J, Bewley AF, Gandour-Edwards RF, Moore MG, Farwell DG, Marcu L. Fluorescence lifetime imaging for intraoperative cancer delineation in transoral robotic surgery. Transl Biophotonics 2019;1. doi: 10.1002/tbio.201900017

45. $\mathrm{Hu} W$, Shen $W$, Zhou H, Kong D. Matrix Linear Discriminant Analysis. Technometrics 2020;62:196-205. 